\title{
Synthesis and Biological Evaluation of 1,2,4-Triazoles and 1,3,4-Oxadiazoles Derivatives Linked to 1,4-Dihydropyridines Scaffold
}

\author{
Maghsoud Ziaie, ${ }^{1}$ Karim Akbari Dilmaghani ${ }^{1, \star}$ and Amir Tukmechi ${ }^{2}$ \\ ${ }^{1}$ Department of Organic Chemistry, Faculty of Chemistry, Urmia University, 57159, Urmia, Iran \\ ${ }^{2}$ Department of Microbiology, Faculty of Veterinary Medicine, Urmia University, Urmia, Iran \\ *Corresponding author: E-mail: kadilmaghani@yahoo.com
}

Received: 06-05-2017

\begin{abstract}
A series of diethyl-2,6-dimethyl-4-phenyl-1,4-dihydropyridine-3,5-dicarboxylate derivative coupled to 1,3,4-oxadiazole-5-thiones and 1,2,4-triazole-5-thiones moieties at C2,C6 positions of 1,4-dihydropyridine ring system was prepared. This linkage was carried out by the reaction of 1,3,4-oxadiazole-5-thiones and 1,2,4-triazole-5-thiones with 2,6-dibromomethyl-3,5-diethoxycarbonyl-4-phenyl-1,4-dihydropyridine in the presence of potassium carbonate as a weak base and dry acetone as the solvent. The newly synthesized compounds were characterized by FT-IR, ${ }^{1} \mathrm{H}$ NMR, ${ }^{13} \mathrm{C}$ NMR spectral data, elemental analysis and FAB-MS. The synthesized compounds were tested for their antimicrobial and antifungal activity against Escherichia coli and Aspergillus fumigatus in vitro in comparison with Enrofloxacin and Amphotericin as the reference drugs which are normally used for treating such infections. The synthetic compounds showed different inhibition zones against tested bacteria and fungi. Compound $\mathbf{8 d}$ showed more antagonistic activity against $E$. coli and A. fumigatus.
\end{abstract}

Key words: 1,4-dihydropyridines, 1,3,4-oxadiazoles, 1,2,4-triazoles, antimicrobial activity

\section{Introduction}

Antibiotics are drugs used for treating infection caused by microorganism such as bacteria or fungi and antibiotic resistance is the ability of microorganism to withstand the effect of antibiotics. The resistance of infective bacteria to present antibiotics remains a clinical obstacle in the chemotherapy of many cancers and demands research focused on the discovery of new drugs in the antibiotic drug field. The 1,4-dihydropyridines (1,4-DHPs) were found to be highly effective calcium antagonists and are used in treatment of various cardiovascular activities. ${ }^{1-4}$ Apart from CVS activities (1,4-DHPs), they posses a variety of biological activities such as anti-tubercular, ${ }^{5}$ antimicrobial, ${ }^{6-7}$ anti-inflammatory, ${ }^{8}$ anti-tumor, ${ }^{9-10}$ analgesic. ${ }^{11}$ A substance which has no calcium antagonistic activity but has antibiotic activity would be of value in cancer chemotherapy and has strong ability in overcoming anticancer drug resistance. Among the possible drug resistance modifiers the 1,4-dihydropyridines calcium antagonists such as Verapamil have been extensively studied. ${ }^{12-13}$ These examples clearly demonstrate the remarkable potential of novel DHP derivatives as a source of valuable drug candidates.

In recent years 1,3,4-oxadiazole-5-thiones and 1,2,4-triazole-5-thiones derivatives which belong to an important group of heterocyclic compounds containing five membered ring have received significant attention and numerous reports have highlighted their diverse range of biological activities, such as antimicrobial, ${ }^{14-15}$ antifungal, ${ }^{16-17}$ antimycobacterial, ${ }^{18-19}$ anticancer, ${ }^{20-21}$ anti-inflammatory $^{22-23}$ and anti convulsant. ${ }^{24-25}$ The connection of 1,3,4-oxadiazoles or 1,2,4-triazoles to the 1,4-DHPs core has produced a combination scaffold. 1,4-DHPs can be selectively functionalized at several positions. Synthesis and antibacterial activities of bis(1,3,4-oxadiazole-2-thiol) and bis(4amino-1,2,4-triazole-3-thiole) derivatives of 1,4-dihdropyridines derivatized at C3, C5 was reported. ${ }^{26}$ Synthesis and biological activities of 1,3,4-oxadiazole derivatives linked to $\mathrm{N} 1$ of 1,4-dihydropyridines ring system was reported. ${ }^{27}$ In spite of the widely developed chemistry of the 1,4-DHPs, much less is known about the synthesis of 1,4-DHPs bearing- substituents other than hydrogen atoms or alkyl groups 
at $\mathrm{C} 2$ and C6. These multicompound 1,4-DHP derivatives are useful in probing biological activity and might be used as antibiotics and inhibitors of the multidrug-resistant transporters. 1,4-DHP derivatives were prepared by Hantzsch method. ${ }^{28}$ Hantzsh dihydropyridine synthesis is a one-pot multicomponent condensation reaction where an aldehyde $\beta$-ketoester and a nitrogen donor, such as ammonia or ammonium acetate, under reflux in ethanol froms the final product. ${ }^{29}$ In our previous works we reported the synthesis and antibacterial properties of a new series of thioglycoside heterocyclic derivatives of 1,2,4-triazole-5 -thiones and 1,3,4-oxadiazole-5-thiones. ${ }^{30-32}$ Following our studies on the design of potent antibacterial agents with 1,3,4-oxadiazoles and 1,2,4-triazole moieties, we have reported the connection of 1,2,4-triazole-5-thiones and 1,3,4-oxadiazole-5-thiones moieties to the 2,6-position of 1,4-DHP ring system. These newly synthesized multicompounds 1,4-DHPs are useful in probing biological activity, as they could exhibit antimicrobial effects and could act as inhibitors of the multidrug-resistant transporters.

\section{Materials and Methods}

\section{1. Bacterial Strain}

The antibacterial and antifungal activity of compounds was assayed according to our perviously published method. ${ }^{33}$ The antibacterial and antifungal activity of compounds was tested against Escherichia coli and Aspergillus fumigatus.

\section{2 Bacterium and Fungi Culture}

The following microorganisms were used in this study to test antimicrobial activity of compounds: E. coli (PTCC 1399) and Aspergillus fumigatus (PTCC 5009). All microorganisms were provided by Persian Culture Collections of Microorganism, Iran. Bacteria were cultured for $24 \mathrm{~h}$ at $37^{\circ} \mathrm{C}$ in brain heart infusion broth (Merck, Darmstadt, Germany) and A. fumigatus was cultured for $72 \mathrm{~h}$ at $5{ }^{\circ} \mathrm{C}$ in Saburo Dextrose broth (Merck, Darmstadt, Germany) and were used as inoculums.

\section{3. Susceptibility Tests}

The following methods were used to evaluate the activity of the compounds. All tests were repeated three times, using distilled water without compounds as a control to test the inhibitory effect of the solvent. Minimum inhibition concentrations (MIC) of compounds against the tested pathological microorganisms were determined using micro broth dilution method. ${ }^{34}$ Briefly, serial twofold dilutions of each compounds $(10 \% \mathrm{w} / \mathrm{v})$ were prepared in 96-well micro titer plate ((from 1:2 to 1:8192) containing cation-adjusted Mueller-Hinton broth (Merck, Darmstadt, Germany). Control micro-titer plates containing medium and distilled water at the same dilutions were also made. Bacteria and fungi suspensions were adjusted to the $0.5 \mathrm{McF}$ arland standards (approximately 1 to $2 \times$ $108 \mathrm{CFU} / \mathrm{mL}$ ). A constant amount of microorganisms were added to all wells and the plates were incubated at 37 and $25^{\circ} \mathrm{C}$ for $24-72$ hour for E. coli and A. fumigatus, respectively (final inoculates were adjusted to the $10^{5} \mathrm{CFU}$ per each well). Each well was examined for growth, comparing each well to the control. The MIC was defined as the lowest concentration of compounds at which there was no visible growth of the organisms. For each test enrofloxacin and amphotericin were used as the control antimicrobial agents. The minimal bactericidal concentration (MBC; the lowest concentration of compounds that resulted in a 99.9\% reduction in CFU of the initial inoculums) was determined by plating count the contents of wells that showed no visible growth of bacteria onto Mueller-Hinton agar and Saburo Dextrose agar plates and incubating at 37 and $25^{\circ} \mathrm{C}$ for $24-72 \mathrm{~h}$ for E. coli and A. fumigatus, respectively. The MBC was considered the lowest concentration of compounds that prevented any colony formation.

\section{Result and Discussion}

The main synthetic route for substituted 1,3,4-oxadiazole-5-thiones $\mathbf{2 a - d}$ involves an initial reaction between carboxylic acid hydrazides 1a-d and carbon disulfide in basic ethanol solution, followed by acidification with di-

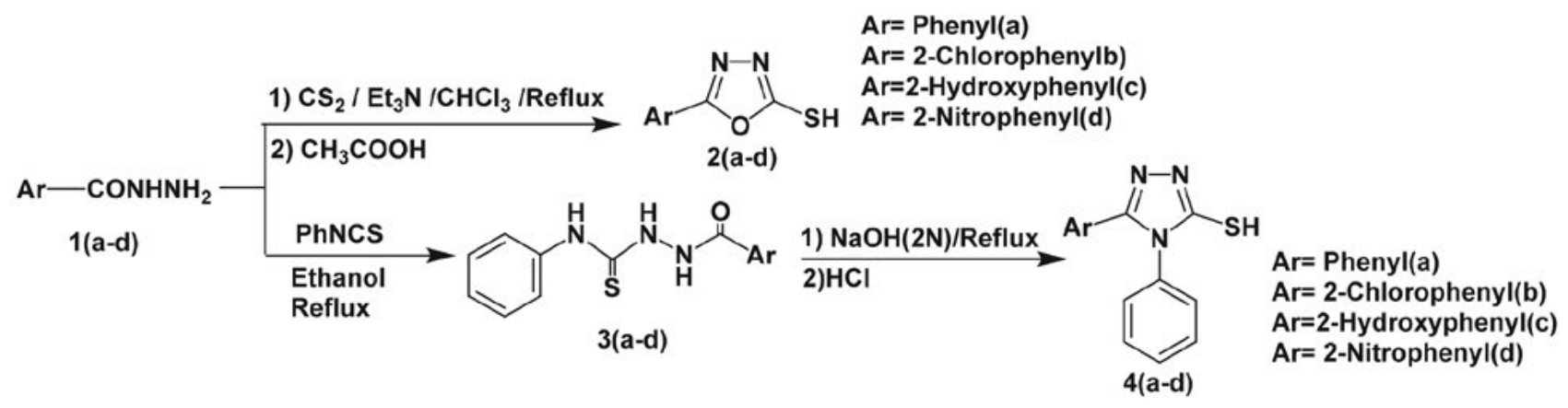

Scheme 1. Synthesis of 1,3,4-oxadiazole-5-thione and 1,2,4-triazole-5-thione derivatives 
lute hydrochloric acid which resulted in the precipitation of oxadiazole. These compounds were synthesized according to the literature method (Scheme 1). ${ }^{35-36} 1$-Substituted-4-phenylthiosemicarbazides $\mathbf{3 a - d}$ were prepared by the condensation of carboxylic acid hydrazides 1a-d with phenylisothiocyanate under reflux in absolute ethanol. The cyclodehydration of 1-substituted-4-phenylthiosemicarbazides 3a-d were carried out in alkaline medium followed by acidification with dilute hydrochloric acid to give 1,2,4-triazole-5-thiones $\mathbf{4 a - d}$ according to the literature method (Scheme 1). ${ }^{37-39}$ The existence of thiol-thione tautomerism is known for the compounds $\mathbf{2 a}-\mathbf{d}$ and $\mathbf{4 a}-\mathbf{d}$ and generally one form is predominant. In the present study, the thione structure was dominated in the solid state. These constitutional isomers were distinguished by IR and ${ }^{1} \mathrm{H}$ NMR. The appearance of a $\mathrm{C}=\mathrm{S}$ absorption peak in the region $1248-1278 \mathrm{~cm}^{-1}$ indicated that the oxadiazoles and triazoles are in their thione form. The ${ }^{1} \mathrm{H}$ NMR spectra of compounds $\mathbf{2 a}-\mathbf{d}$ and $\mathbf{4 a}-\mathbf{d}$ exhibited the $\mathrm{NH}$ signals $(\mathrm{NH}$ function of the oxadiazole and triazole ring) as a singlet in the range $\delta_{\mathrm{H}} 12-14 \mathrm{ppm}$ which also supports the proposed thione structure. ${ }^{35-39}$

4-Phenyl-1,4-dihydro-2,6-dimethyl-3,5-pyridine dicarboxylic acid ester (diethyl 2,6-dimethyl-4-phenyl1,4-dihydropyridine-3,5-dicarboxylate)derivative (compound 5) was synthesized by condensation of benzaldehyde with two equivalents of $\beta$-ketoester in the presence of a nitrogen donor such as ammonia or ammonium acetate according to the procedure reported in the literature. ${ }^{40}$ The ${ }^{1} \mathrm{H}$ NMR spectrum of compound 5 shows a characteristic singlet in the range of $\delta_{\mathrm{H}} 5-7 \mathrm{ppm}$ which was due to the $\mathrm{NH}$ proton of the 1,4-DHP ring and another important singlet at $5 \mathrm{ppm}$ which was attributed to the $\mathrm{CH}$ at $\mathrm{C} 4$ of the 1,4-DHP ring. Allylic bromination is the replacement of a hydrogen on a carbon adjacent to a double bond and $\mathrm{N}$-bromosuccinimide (NBS) is a brominating agent that is used as the source of bromine in radical reactions that are used for allylic bromination. The synthesis of 2,6-dibromomethyl-3,5-diethoxycarbonyl-1,4-dihydropyridine (compound 6) was carried out by bromination of corresponding 2,6-dimethyl-1,4-dihydropyridine (compound 5) with NBS in methanol according to the procedure reported in the literature ${ }^{41}$ Replacement of the bromines of compound 6 with 1,2,4-triazole-5-thiones $4 \mathbf{a}-\mathbf{d}$ or 1,3,4oxadiazole-5-thiones $2 \mathbf{a}-\mathbf{d}$ was carried out in the presence of potassium carbonate as a weak base in dry acetone to afford the corresponding coupled 1,4-dihydropyridines (7a-d and 8a-d) (Scheme 2). The structures identification for compounds $7 \mathbf{a}-\mathbf{d}$ and $\mathbf{8 a}-\mathbf{d}$ was based on spectroscopic methods. In the IR spectra the disappearance of the $\mathrm{C}=\mathrm{S}$ absorption peak in the region $1248-1278 \mathrm{~cm}^{-1}$ and furthermore the absence of $\mathrm{NH}$ peak at 12-14 ppm support the connection of oxadiazole and triazoles to 1,4-DHP ring.

The $\mathrm{CH}_{2} \mathrm{X}$ protons at positions $\mathrm{C} 2$ and $\mathrm{C} 6$ of symmetrically substituted 1,4-dihydropyridine ring become diastereotopic and provide an $\mathrm{AB}$ system in the corresponding ${ }^{1} \mathrm{H}$ NMR spectra. The extent of the observed anisochrony of the methylene protons should be influenced by the spatial conformation of ester groups and the formation of a $\mathrm{CH} \cdots \mathrm{O}=\mathrm{C}$ intramolecular hydrogen bonding. ${ }^{41}$

The in vitro antibacterial and antifungal activities of the synthesized compounds against E. coli and A. fumigatus are shown in Tables 1 and 2. The minimal inhibition concentration for enrofloxacin as the reference antibacterial drug is $36 \mu \mathrm{g} / \mu \mathrm{L}$ concentration and for amphotericin was $28 \mu \mathrm{g} / \mu \mathrm{L}$ of drug in the same test conditions.

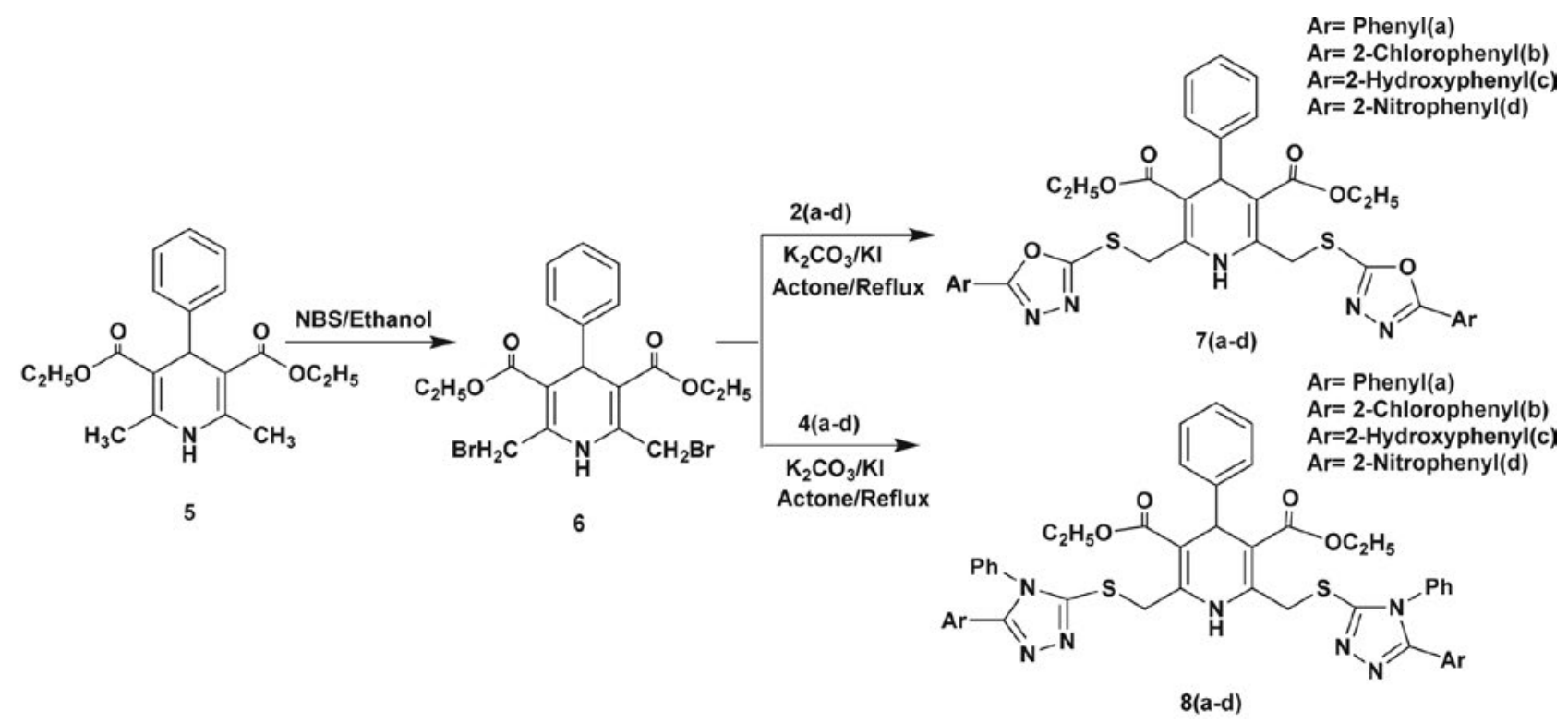

Scheme 2. Synthesis of 1,4-dihydropyridine compounds bearing 1,3,4-oxadiazole-5-thiones and 1,2,4-triazole-5-thiones 
Table 1. In vitro antibacterial activity of synthetic compounds against E. coli.

\begin{tabular}{lccccc}
\hline Compound & $\begin{array}{c}\text { MIC } \\
(\mathbf{g} / \mathbf{m L})\end{array}$ & $\begin{array}{c}\text { MBC } \\
(\mathbf{g} / \mathbf{m L})\end{array}$ & Compound & $\begin{array}{c}\text { MIC } \\
(\mathbf{g} / \mathbf{m L})\end{array}$ & $\begin{array}{c}\text { MBC } \\
(\mathbf{g} / \mathbf{m L})\end{array}$ \\
\hline $\mathbf{8 a}$ & 250 & 500 & $\mathbf{7 a}$ & 500 & 500 \\
$\mathbf{8 b}$ & 250 & 500 & $\mathbf{7 b}$ & 125 & 125 \\
$\mathbf{8 c}$ & 125 & 250 & $\mathbf{7 c}$ & 62.5 & 125 \\
$\mathbf{8 d}$ & 62.5 & 62.5 & $\mathbf{7 d}$ & 62.5 & 125 \\
\hline
\end{tabular}

Table 2. In vitro antifungal activity of synthetic compounds against A. fumigatus.

\begin{tabular}{lccccc}
\hline Compound & $\begin{array}{c}\text { MIC } \\
(\mathbf{g} / \mathbf{m L})\end{array}$ & $\begin{array}{c}\text { MFC } \\
(\mathbf{g} / \mathbf{m L})\end{array}$ & Compound & $\begin{array}{c}\text { MIC } \\
(\mathbf{g} / \mathbf{m L})\end{array}$ & $\begin{array}{c}\text { MFC } \\
(\mathbf{g} / \mathbf{m L})\end{array}$ \\
\hline $\mathbf{8 a}$ & 500 & 500 & $\mathbf{7 a}$ & 1000 & 2000 \\
$\mathbf{8 b}$ & 500 & 500 & $\mathbf{7 b}$ & 1000 & 2000 \\
$\mathbf{8 c}$ & 500 & 500 & $\mathbf{7 c}$ & 1000 & 2000 \\
$\mathbf{8 d}$ & 250 & 500 & $\mathbf{7 d}$ & 500 & 1000 \\
\hline
\end{tabular}

From the data presented in Tables 1 and 2 it is clear that compounds $\mathbf{8 a}-\mathbf{d}$ showed better antifungal activities than compounds $\mathbf{7 a - d}$ and the compounds $\mathbf{7 c}, 7 \mathbf{d}, \mathbf{8 d}$ exhibited better and equipotent activity against $E$. coli. The compound $\mathbf{8 d}$ is highly active against $E$. coli and A. fumigatus.

\section{Experimental}

\section{1. General}

The melting points of all compounds were recorded on a Philip Harris C4954718 apparatus without calibration. IR spectra were recorded on Thermo Nicolet Nexus 670 FT-IR spectrometer and ${ }^{1} \mathrm{H}$ and ${ }^{13} \mathrm{C}$ NMR spectra measured with Bruker Avance $300 \mathrm{MHz}$ spectrometer. Mass spectra were recorded on a JEOL-JMS 600 (FAB MS) instrument. Thin layer chromatography (TLC) analyses were carried out on silica gel plates. All chemicals were purchased from Merck (Tehran, Iran) and used as received by standard procedures. All of the instruments, chemicals and solvents were dried according to standard methods. Freshly distilled solvents were used throughout, and anhydrous solvents were dried according to the method reported by Perrin and Armarego. Microanalyses were performed on a Leco Analyzer 932.

\section{2. General Procedure for the Synthesis of $2 a-d$}

Compounds 2a-d were synthesized by the reaction of $\mathrm{CS}_{2}(6.7 \mathrm{~mL}, 0.1 \mathrm{~mol})$ with a suspension of carboxylic acid hydrazides 1a-d $(0.1 \mathrm{~mol})$ in chloroform $(100 \mathrm{~mL})$ in the presence of $\mathrm{Et}_{3} \mathrm{~N}(15 \mathrm{~mL}, 0.1 \mathrm{~mol})$. After refluxing the reaction mixture for $3 \mathrm{~h}$, the resulting solution was filtered and acidified with acetic acid $(20 \mathrm{~mL}, 20 \% \mathrm{v} / \mathrm{v})$. On evaporation of the solvent, the solid obtained was washed twice with cold water and finally dried in vacuo, then recrystallized from ethanol (yield $80 \%$ ). ${ }^{35-36}$

\section{3. General Procedure for the Synthesis of $4 a-d$}

Equimolar quantities of carboxylic acid hydrazides 1a-d $(0.01 \mathrm{~mol})$ and phenylisothiocyanate $(1.35 \mathrm{~g}, 0.01$ $\mathrm{mol})$ in appropriate amount of absolute ethanol were refluxed for 6-8 h. The formed precipitate of 1-substituted-4-phenylthiosemicarbazides $\mathbf{3 a}-\mathbf{d}$ was filtered, and then a suspension of $0.04 \mathrm{~g}$ thiosemicarbazides $\mathbf{3 a} \mathbf{a}-\mathbf{d}$ in sodium hydroxide $0.4 \mathrm{~g}(0.01 \mathrm{~mol}$, as a $2 \mathrm{~N}$ solution $)$ was refluxed for $6 \mathrm{~h}$.

The reaction mixture was allowed to cool to the room temperature and was then adjusted to $\mathrm{pH} 6$ with diluted hydrochloric acid. The formed precipitate was then filtered, dried and recrystallized from ethanol, to give 1,2,4-triazole-5-thiones $\mathbf{4 a - d}$ in 75\% yields. The following compounds were prepared by an analogous procedure. ${ }^{37-39}$

Diethyl 2,6-Dimethyl-4-phenyl-1,4-dihydropyridine-3, 5-dicarboxylate $(5)^{40}$

White crystals; $77 \%(2.535 \mathrm{~g}), \mathrm{mp} 160-162{ }^{\circ} \mathrm{C},{ }^{1} \mathrm{H}$ NMR $\left(300 \mathrm{MHz}, \mathrm{CDCl}_{3}\right) \delta 1.25(\mathrm{t}, J=7.2 \mathrm{~Hz}, 6 \mathrm{H}$, $\left.-\mathrm{COOCH}_{2} \mathrm{CH}_{3}\right), 2.35\left(\mathrm{~s}, 6 \mathrm{H}, 2 \mathrm{CH}_{3}\right), 4.10(\mathrm{q}, 4 \mathrm{H}$, $\left.-\mathrm{COOCH}_{2} \mathrm{CH}_{3}\right), 5.02\left(\mathrm{~s}, 1 \mathrm{H}, \mathrm{DHP}-\mathrm{C}_{4}-\mathrm{H}\right), 5.72(\mathrm{br} \mathrm{s}, 1 \mathrm{H}$, $\mathrm{N}-\mathrm{H}), 7.13-7.32(\mathrm{~m}, 5 \mathrm{H}, \mathrm{Ar}-\mathrm{H}),{ }^{13} \mathrm{C}$ NMR $(75 \mathrm{MHz}$, $\left.\mathrm{CDCl}_{3}\right) \delta 14.27,19.12,39.66,59.75,103.59,126.11,127.85$, $127.92,144.76,147.96,168.03$. IR (KBr) v 3340, 3078, 2976, 1651, 1483, 1378, 1100, 1030, 766, $695 \mathrm{~cm}^{-1}$. Anal. calcd for $\mathrm{C}_{19} \mathrm{H}_{23} \mathrm{NO}_{4}: \mathrm{C}, 69.28 ; \mathrm{H}, 7.04 ; \mathrm{N}, 4.25$. Found: C, $69.18 ; \mathrm{H}, 6.98 ; \mathrm{N}, 4.29$.

Diethyl 2,6-Bis(bromomethyl)-4-phenyl-1,4-dihydropyridine-3,5-dicarboxylate (6) ${ }^{41}$

Lemon crystals; $72 \%$ (3.506 g), mp $135^{\circ} \mathrm{C},{ }^{1} \mathrm{H}$ NMR $\left(300 \mathrm{MHz}, \mathrm{CDCl}_{3}\right) \delta 1.25\left(\mathrm{t}, J=7.2 \mathrm{~Hz}, 6 \mathrm{H},-\mathrm{COOCH}_{2} \mathrm{CH}_{3}\right)$, $4.15\left(\mathrm{q}, J=7.2 \mathrm{~Hz}, 4 \mathrm{H},-\mathrm{COOCH}_{2} \mathrm{CH}_{3}\right), 4.6,4.9\left(\mathrm{dd}, J_{1}=\right.$ $\left.4.65, J_{2}=4.65 \mathrm{~Hz}, 4 \mathrm{H}, \mathrm{CH}_{2}-\mathrm{Br}\right), 5.03\left(\mathrm{~s}, 1 \mathrm{H}, \mathrm{DHP}_{-} \mathrm{C}_{4}-\mathrm{H}\right)$, 6.53 (br s,1H, N-H), 7.12-7.31 (m, 5H, Ar-H), ${ }^{13} \mathrm{C}$ NMR $\left(75 \mathrm{MHz}, \mathrm{CDCl}_{3}\right) \delta 14.11,27.15,40.08,60.65,105.79$, $126.84,128.06,128.19,142.19,145.85,166.31$. IR (KBr) v 3334, 3084, 2979, 1686, 1508, 1372, 1095, 760, 704, 558 $\mathrm{cm}^{-1}$. Anal. calcd for $\mathrm{C}_{19} \mathrm{H}_{21} \mathrm{Br}_{2} \mathrm{NO}_{4}: \mathrm{C}, 46.84 ; \mathrm{H}, 4.34 ; \mathrm{N}$, 2.88. Found: C, 46.76; H, 4.31; N, 2.95 .

\section{4. General Procedure for the Synthesis of Compounds 7a-d and 8a-d}

A mixture of $\mathbf{2 a}-\mathbf{d}$ or $4 \mathbf{a}-\mathbf{d}(2 \mathrm{mmol}), \mathrm{K}_{2} \mathrm{CO}_{3}(0.276 \mathrm{~g}$, $2 \mathrm{mmol})$ and acetone $(20 \mathrm{~mL})$ were stirred at room temperature for 30 minute, and then $0.487 \mathrm{~g}(1 \mathrm{mmol}) \mathrm{com}$ - 
pound 6 and KI (cat.) were added and refluxed for $12 \mathrm{~h}$ and then evaporated. The residue was extracted with $\mathrm{CH}_{2} \mathrm{Cl}_{2}$ $(30 \mathrm{~mL})$ and the organic layer washed with water, dried over $\mathrm{Na}_{2} \mathrm{SO}_{4}$ and evaporated. Recrystallization of crude products from 2-propanol furnished $7 \mathbf{a}-\mathbf{d}$ or $\mathbf{8 a}-\mathbf{d}$.

Diethyl 4-Phenyl-2, 6-bis(((5-phenyl-1,3,4-oxadiazol-2yl)thio)methyl)-1,4-dihydropyridine-3,5-dicarboxylate (7a)

Pale yellow solid; $73 \%\left(0.498\right.$ g), m.p. $156-158^{\circ} \mathrm{C},{ }^{1} \mathrm{H}$ NMR $\left(300 \mathrm{MHz}, \mathrm{CDCl}_{3}\right): \delta 25(\mathrm{t}, J=7.2 \mathrm{~Hz}, 6 \mathrm{H}$, $\left.-\mathrm{COOCH}_{2} \mathrm{CH}_{3}\right), 4.14\left(\mathrm{q}, J=7.2 \mathrm{~Hz}, 4 \mathrm{H},-\mathrm{COOCH}_{2} \mathrm{CH}_{3}\right)$, $4.62\left(\mathrm{~s}, 4 \mathrm{H}, \mathrm{S}-\mathrm{CH}_{2}\right), 5.05\left(\mathrm{~s}, 1 \mathrm{H}, \mathrm{DHP}_{-} \mathrm{C}_{4}-\mathrm{H}\right), 7.11-7.31$ (m, 5H, Ar-H), 7.40-7.52 (m, 6H, Ar-H), $7.90(\mathrm{~d}, J=$ $8.1 \mathrm{~Hz}, 4 \mathrm{H}, \mathrm{Ar}-\mathrm{H}), 8.78$ (br s, $1 \mathrm{H}, \mathrm{N}-\mathrm{H}),{ }^{13} \mathrm{C}$ NMR $(75$ $\left.\mathrm{MHz}, \mathrm{CDCl}_{3}\right) \delta 14.13,32.32,39.66,60.37,104.99,123.54$, $126.62,126.66,126.71,128.11,128.94,131.59,144.17$, $147.76,162.32,165.38,166.88 . \mathrm{IR}(\mathrm{KBr}) \vee 3330,3073,2979$, $1688,1636,1483,1375,1090,764,695,595 \mathrm{~cm}^{-1}$. Anal. calcd for $\mathrm{C}_{35} \mathrm{H}_{31} \mathrm{~N}_{5} \mathrm{O}_{6} \mathrm{~S}_{2}: \mathrm{C} 61.66, \mathrm{H} 4.58, \mathrm{~N} 10.27, \mathrm{~S} 9.40$. Found: C, 61.69; H, 4.53; N, 10.24; S, 9.47. HRMS-FAB $\mathrm{m} / z[\mathrm{M}+\mathrm{H}]^{+}$calcd for $\mathrm{C}_{35} \mathrm{H}_{31} \mathrm{~N}_{5} \mathrm{O}_{6} \mathrm{~S}_{2}:$ 681.17. Found: 681.11 .

Diethyl 2,6-Bis((5-(2-chlorophenyl)-1,3,4-oxadiazol-2ylthio)methyl)-4-phenyl-1,4-dihydropyridine-3,5-dicarboxylate (7b)

Pale yellow solid; $62 \%\left(0.465\right.$ g), m.p. $156-158^{\circ} \mathrm{C},{ }^{1} \mathrm{H}$ NMR $\left(300 \mathrm{MHz}, \mathrm{DMSO}-d_{6}\right) \delta 1.25(\mathrm{t}, J=7.2 \mathrm{~Hz}, 6 \mathrm{H}$, $\left.-\mathrm{COOCH}_{2} \mathrm{CH}_{3}\right), 4.15\left(\mathrm{q}, J=7.2 \mathrm{~Hz}, 4 \mathrm{H},-\mathrm{COOCH}_{2} \mathrm{CH}_{3}\right)$, $4.65\left(\mathrm{dd}, J_{1}=14.1 \mathrm{~Hz}, J_{2}=14.1 \mathrm{~Hz}, 4 \mathrm{H}, \mathrm{S}-\mathrm{CH}_{2}\right), 5.05(\mathrm{~s}, 1 \mathrm{H}$, DHP-C $\left._{4}-\mathrm{H}\right), 7.10-7.32(\mathrm{~m}, 6 \mathrm{H}, \mathrm{Ar}-\mathrm{H}), 7.32-7.51(\mathrm{~m}, 5 \mathrm{H}$, Ar-H), 7.86 (d, J=6.6 Hz, 2H, Ar-H), 8.72 (br s, $1 \mathrm{H}, \mathrm{N}-\mathrm{H})$; ${ }^{13} \mathrm{C}$ NMR $\left(75 \mathrm{MHz}\right.$, DMSO- $\left.d_{6}\right) \delta 14.32,32.83,39.60,60.31$, $104.53,122.53,126.94,127.82,128.19,128.49,131.48$, $131.58,132.22,133.70,44.16,146.99,162.32,164.22$, 166.41. IR (KBr) v 3282, 3080, 2978, 1678, 1630, 1488, 1097, $739 \mathrm{~cm}^{-1}$. HRMS-FAB $\mathrm{m} / z[\mathrm{M}+\mathrm{H}]^{+}$calcd for $\mathrm{C}_{35} \mathrm{H}_{29} \mathrm{Cl}_{2} \mathrm{~N}_{5} \mathrm{O}_{6} \mathrm{~S}_{2}: 749.09$. Found: 749.14. Anal. calcd for $\mathrm{C}_{35} \mathrm{H}_{29} \mathrm{Cl}_{2} \mathrm{~N}_{5} \mathrm{O}_{6} \mathrm{~S}_{2}: \mathrm{C}, 56.00 ; \mathrm{H}, 3.89 ; \mathrm{N}, 9.33 ; \mathrm{S}, 8.54$. Found: C, $56.04 ; \mathrm{H}, 3.84 ; \mathrm{N}, 9.35 ; \mathrm{S}, 8.57$.

Diethyl 2,6-Bis((5-(2-hydroxyphenyl)-1,3,4-oxadiazol2-ylthio)methyl)-4-phenyl-1,4-dihydropyridine-3,5-dicarboxylate $(7 \mathrm{c})$

Pale yellow solid; $69 \%$ (0.492 g), m.p. $155-157^{\circ} \mathrm{C},{ }^{1} \mathrm{H}$ $\mathrm{NMR}\left(300 \mathrm{MHz}, \mathrm{CDCl}_{3}\right) \delta 1.26(\mathrm{t}, J=7.2 \mathrm{~Hz}, 6 \mathrm{H}$, $\left.-\mathrm{COOCH}_{2} \mathrm{CH}_{3}\right), 4.14\left(\mathrm{q}, J=7.2 \mathrm{~Hz}, 4 \mathrm{H},-\mathrm{COOCH}_{2} \mathrm{CH}_{3}\right)$, $4.63\left(\mathrm{dd}, J_{1}=14.1 \mathrm{~Hz}, J_{2}=14.1 \mathrm{~Hz}, 4 \mathrm{H}, \mathrm{S}-\mathrm{CH}_{2}\right), 5.03(\mathrm{~s}, 1 \mathrm{H}$, DHP-C $-\mathrm{H}), 6.95(\mathrm{t}, J=7.5 \mathrm{~Hz}, 2 \mathrm{H}, \mathrm{Ar}-\mathrm{H}), 7.05(\mathrm{~d}, J=8.4$ $\mathrm{Hz}, 2 \mathrm{H}, \mathrm{Ar}-\mathrm{H}), 7.12-7.32(\mathrm{~m}, 5 \mathrm{H}, \mathrm{Ar}-\mathrm{H}), 7.42(\mathrm{t}, J=7.5$ $\mathrm{Hz}, 2 \mathrm{H}, \mathrm{Ar}-\mathrm{H}), 7.65$ (d, J $=7.8 \mathrm{~Hz}, 2 \mathrm{H}, \mathrm{Ar}-\mathrm{H}), 8.59(\mathrm{~s}, 1 \mathrm{H}$, $\mathrm{N}-\mathrm{H}), 9.73$ (s, 2H, OH). ${ }^{13} \mathrm{C}$ NMR $\left(75 \mathrm{MHz}, \mathrm{CDCl}_{3}\right) \delta$ $14.12,32.43,39.63,60.47,105.41,107.66,117.47,119.92$, $126.45,126.73,128.02,128.12,133.66,143.66,146.47$, $157.16,164.75,165.64,166.78$. IR (KBr) v 3302, 2879,
$1692,1620,1486,1100,753,703 \mathrm{~cm}^{-1}$. HRMS-FAB $\mathrm{m} / \mathrm{z}$ $[\mathrm{M}+\mathrm{H}]^{+}$calcd for $\mathrm{C}_{35} \mathrm{H}_{31} \mathrm{~N}_{2} \mathrm{O}_{8} \mathrm{~S}_{2}: 713.16$. Found: 713.23. Anal. calcd for $\mathrm{C}_{35} \mathrm{H}_{31} \mathrm{~N}_{5} \mathrm{O}_{8} \mathrm{~S}_{2}$ : C, 58.90; $\mathrm{H}, 4.38 ; \mathrm{N}, 9.81 ; \mathrm{S}$, 8.98. Found: C, 58.86; H, 4.33; N, 9.84; S, 9.02.

Diethyl 2,6-Bis((5-(3-nitrophenyl)-1,3,4-oxadiazol-2ylthio)methyl)-4-phenyl-1,4-dihydropyridine-3,5-dicarboxylate (7d)

Yellow crystals; $64 \%\left(0.493\right.$ g), m.p. $150-151{ }^{\circ} \mathrm{C},{ }^{1} \mathrm{H}$ NMR $\left(300 \mathrm{MHz}, \mathrm{CDCl}_{3}\right) \delta 1.26(\mathrm{t}, J=7.2 \mathrm{~Hz}, 6 \mathrm{H}$, $\left.-\mathrm{COOCH}_{2} \mathrm{CH}_{3}\right), 4.15\left(\mathrm{q}, J=7.2 \mathrm{~Hz}, 4 \mathrm{H},-\mathrm{COOCH}_{2} \mathrm{CH}_{3}\right)$, 4.65 (s, 4H, S- $\left.\mathrm{CH}_{2}\right), 5.04$ (s, 1H, DHP-C $\left.-\mathrm{H}\right), 7.10-7.30$ (m, 5H, Ar-H, 1H, N-H), $7.69(\mathrm{t}, J=7.8 \mathrm{~Hz}, 2 \mathrm{H}, \mathrm{Ar}-\mathrm{H})$, $8.28(\mathrm{~d}, J=7.8 \mathrm{~Hz}, 2 \mathrm{H}, \mathrm{Ar}-\mathrm{H}), 8.36(\mathrm{~d}, J=8.1 \mathrm{~Hz}, 2 \mathrm{H}, \mathrm{Ar}-$ $\mathrm{H}), 8.72(\mathrm{~d}, J=17.1 \mathrm{~Hz}, 2 \mathrm{H}, \mathrm{Ar}-\mathrm{H}) .{ }^{13} \mathrm{C}$ NMR $(75 \mathrm{MHz}$, $\left.\mathrm{CDCl}_{3}\right) \delta 14.12,32.35,39.65,60.47,105.25,121.49,125.13$, $126.04,126.66,128.06,128.09,130.38,132.04,143.81$, $146.65,148.60,162.32,164.28,166.85$. IR (KBr) v 3333 , 3087, 2978, 1693, 1633, 1525, 1479, 1353, 1097, $710 \mathrm{~cm}^{-1}$. HRMS-FAB $\mathrm{m} / z[\mathrm{M}+\mathrm{H}]^{+}$calcd for $\mathrm{C}_{35} \mathrm{H}_{29} \mathrm{~N}_{7} \mathrm{O}_{10} \mathrm{~S}_{2}: 771.14$. Found: 771.11. Anal. calcd for $\mathrm{C}_{35} \mathrm{H}_{29} \mathrm{~N}_{7} \mathrm{O}_{10} \mathrm{~S}_{2}: \mathrm{C}, 54.47 ; \mathrm{H}$, 3.79; N, 12.70; S, 8.31. Found: C, 54.51; H, 3.76; N, 12.67; S, 8.36 .

Diethyl 2,6-Bis((4,5-diphenyl-4H-1,2,4-triazol-3-ylthio)methyl)-4-phenyl-1,4-dihydropyridine-3,5-dicarboxylate $(8 \mathrm{a})$

White solid; $76 \%\left(0.632\right.$ g), m.p. ${ }^{189-191}{ }^{\circ} \mathrm{C},{ }^{1} \mathrm{H}$ NMR $\left(300 \mathrm{MHz}, \mathrm{CDCl}_{3}\right) \delta 1.22(\mathrm{t}, J=7.2 \mathrm{~Hz}, 6 \mathrm{H}$, $\left.-\mathrm{COOCH}_{2} \mathrm{CH}_{3}\right), 4.08\left(\mathrm{q}, J=7.2 \mathrm{~Hz}, 4 \mathrm{H},-\mathrm{COOCH}_{2} \mathrm{CH}_{3}\right)$, $4.49,4.66\left(\mathrm{dd}, J_{1}=14.1 \mathrm{~Hz}, J_{2}=14.1 \mathrm{~Hz}, 4 \mathrm{H}, \mathrm{S}_{-} \mathrm{CH}_{2}\right), 5.04$ (s, 1H, DHP-C $-\mathrm{H}), 7.10-7.50(\mathrm{~m}, 25 \mathrm{H}, \mathrm{Ar}-\mathrm{H}), 9.69(\mathrm{~s}, 1 \mathrm{H}$, $\mathrm{N}-\mathrm{H}) .{ }^{13} \mathrm{C}$ NMR $\left(75 \mathrm{MHz}, \mathrm{CDCl}_{3}\right) \delta 14.17,32.35,39.82$, $60.03,104.42,126.30,126.68,127.49,127.94,128.20$, $128.33,129.78,129.84,134.25,145.36,147.32,153.61$, 155.05, 167.11. IR (KBr) v 3292, 3050, 2976, 1688, 1635, $1493,1095,766,695 \mathrm{~cm}^{-1}$. HRMS-FAB $\mathrm{m} / z[\mathrm{M}+\mathrm{H}]^{+}$calcd for $\mathrm{C}_{47} \mathrm{H}_{41} \mathrm{~N}_{7} \mathrm{O}_{4} \mathrm{~S}_{2}: 831.27$. Found: 831.22. Anal. calcd for $\mathrm{C}_{47} \mathrm{H}_{41} \mathrm{~N}_{7} \mathrm{O}_{4} \mathrm{~S}_{2}: \mathrm{C}, 67.85 ; \mathrm{H}, 4.97 ; \mathrm{N}, 11.78 ; \mathrm{S}, 7.71$. Found: C, 67.88; H, 4.94; N, 11.79; S, 7.73.

Diethyl 2,6-Bis((5-(2-chlorophenyl)-4-phenyl-4H-1,2,4 -triazol-3-ylthio)methyl)-4-phenyl-1,4-dihydropyridine-3,5-dicarboxylate ( $8 b$ )

White solid; 61\% (0.549 g), m.p. $164-165{ }^{\circ} \mathrm{C},{ }^{1} \mathrm{H}$ NMR $\left(300 \mathrm{MHz}, \mathrm{CDCl}_{3}\right) \delta 1.22(\mathrm{t}, J=7.2 \mathrm{~Hz}, 6 \mathrm{H}$, $\left.-\mathrm{COOCH}_{2} \mathrm{CH}_{3}\right), 4.09\left(\mathrm{q}, J=7.2 \mathrm{~Hz}, 4 \mathrm{H},-\mathrm{COOCH}_{2} \mathrm{CH}_{3}\right.$ ), $4.52,4.66\left(\mathrm{dd}, J_{1}=14.1 \mathrm{~Hz}, J_{2}=14.1 \mathrm{~Hz}, 4 \mathrm{H}, \mathrm{S}-\mathrm{CH}_{2}\right), 5.04$ (s, 1H, DHP-C $-\mathrm{H}), 7.11-7.36(\mathrm{~m}, 21 \mathrm{H}, \mathrm{Ar}-\mathrm{H}), 7.48(\mathrm{~d}, J=$ $7.2 \mathrm{~Hz}, 2 \mathrm{H}, \mathrm{Ar}-\mathrm{H}), 9.70$ (s, $1 \mathrm{H}, \mathrm{N}-\mathrm{H}) .{ }^{13} \mathrm{C}$ NMR $(75 \mathrm{MHz}$, $\left.\mathrm{CDCl}_{3}\right) \delta 14.17,32.35,39.83,60.08,104.12,126.32,126.61$, $126.78,127.95,128.23,129.33,129.62,131.42,132.79$, $133.34,134.28,145.34,147.25,152.95,153.53,162.32$, 167.12. IR $(\mathrm{KBr}) \vee 3277,3068,2978,1685,1637,1499$, 1094, 763, 697, $604 \mathrm{~cm}^{-1}$; HRMS-FAB $\mathrm{m} / z[\mathrm{M}+\mathrm{H}]^{+}$calcd for $\mathrm{C}_{47} \mathrm{H}_{39} \mathrm{Cl}_{2} \mathrm{~N}_{7} \mathrm{O}_{4} \mathrm{~S}_{2}: 899.19$. Found: 899.25. Anal. calcd 
for $\mathrm{C}_{47} \mathrm{H}_{39} \mathrm{Cl}_{2} \mathrm{~N}_{7} \mathrm{O}_{4} \mathrm{~S}_{2}$ : C, 62.66; H, 4.36; N, 10.88; S, 7.12. Found: C, 62.67; H, 4.35; N, 10.90; S, 7.15.

Diethyl 2,6-Bis((5-(2-hydroxyphenyl)-4-phenyl-4H1,2,4-triazol-3-ylthio)methyl)-4-phenyl-1,4-dihydropyridine-3,5-dicarboxylate $(8 \mathrm{c})$

Pale yellow solid; $71 \%(0.613 \mathrm{~g})$, m.p. $226-228^{\circ} \mathrm{C},{ }^{1} \mathrm{H}$ NMR $\left(300 \mathrm{MHz}, \mathrm{CDCl}_{3}\right) \delta 1.22(\mathrm{t}, J=7.2 \mathrm{~Hz}, 6 \mathrm{H}$, $\left.-\mathrm{COOCH}_{2} \mathrm{CH}_{3}\right), 4.08\left(\mathrm{q}, J=7.2 \mathrm{~Hz}, 4 \mathrm{H},-\mathrm{COOCH}_{2} \mathrm{CH}_{3}\right.$ ), $4.52,4.70\left(\mathrm{dd}, J_{1}=14.1 \mathrm{~Hz}, J_{2}=14.1 \mathrm{~Hz}, 4 \mathrm{H}, \mathrm{S}-\mathrm{CH}_{2}\right), 5.05$ (s, 1H, DHP-C $-\mathrm{H}), 6.40-6.54(\mathrm{~m}, 5 \mathrm{H}, \mathrm{Ar}-\mathrm{H}), 6.97$ (d, $J=$ $8.4 \mathrm{~Hz}, 2 \mathrm{H}, \mathrm{Ar}-\mathrm{H}), 7.12(\mathrm{t}, J=6.9 \mathrm{~Hz}, 2 \mathrm{H}, \mathrm{Ar}-\mathrm{H}), 7.22(\mathrm{t}, J$ $=7.2 \mathrm{~Hz}, 2 \mathrm{H}, \mathrm{Ar}-\mathrm{H}), 7.27-7.37(\mathrm{~m}, 6 \mathrm{H}, \mathrm{Ar}-\mathrm{H}), 7.52-7.63$ (m, 6H, Ar-H), 9.25 (s, 1H, N-H), 11.60 (br s, $2 \mathrm{H}, \mathrm{OH}) .{ }^{13} \mathrm{C}$ NMR $\left(75 \mathrm{MHz}, \mathrm{CDCl}_{3}\right) \delta 14.12,32.13,39.74,60.15$, $104.04,110.04,117.78,118.42,125.48,126.46,127.65$, $127.97,128.16,130.43,130.67,131.27,134.23,144.61$, $147.00,153.68,153.83,157.86,166.97 . \mathrm{IR}(\mathrm{KBr})$ v 3303, 2978, 1691, 1618, 1100, 752, $701 \mathrm{~cm}^{-1}$. HRMS-FAB $\mathrm{m} / \mathrm{z}$ $[\mathrm{M}+\mathrm{H}]^{+}$calcd for $\mathrm{C}_{47} \mathrm{H}_{41} \mathrm{~N}_{7} \mathrm{O}_{6} \mathrm{~S}_{2}: 863.26$. Found: 863.30. Anal. calcd for $\mathrm{C}_{47} \mathrm{H}_{41} \mathrm{~N}_{7} \mathrm{O}_{6} \mathrm{~S}$ : C, 65.34; $\mathrm{H}, 4.78 ; \mathrm{N}, 11.35 ; \mathrm{S}$, 7.42. Found: C, $65.33 ; \mathrm{H}, 4.81 ; \mathrm{N}, 11.32 ; \mathrm{S}, 7.40$.

Diethyl 2,6-Bis((5-(3-nitrophenyl)-4-phenyl-4H-1,2,4triazol-3-ylthio)methyl)-4-phenyl-1,4-dihydropyridine-3,5-dicarboxylate (8d)

Pale yellow crystals; $68 \%\left(0.627\right.$ g), m.p. $189-190{ }^{\circ} \mathrm{C}$, ${ }^{1} \mathrm{H}$ NMR $\left(300 \mathrm{MHz}, \mathrm{CDCl}_{3}\right) \delta 1.25(\mathrm{t}, J=7.2 \mathrm{~Hz}, 6 \mathrm{H}$, $\left.-\mathrm{COOCH}_{2} \mathrm{CH}_{3}\right), 4.08\left(\mathrm{q}, J=7.2 \mathrm{~Hz}, 4 \mathrm{H},-\mathrm{COOCH}_{2} \mathrm{CH}_{3}\right.$ ), $4.56,4.66\left(\mathrm{dd}, J_{1}=14.1 \mathrm{~Hz}, J_{2}=14.1 \mathrm{~Hz}, 4 \mathrm{H}, \mathrm{S}_{-}-\mathrm{CH}_{2}\right), 5.04(\mathrm{~s}$, 1H, DHP-C $-\mathrm{H}), 7.17-7.58$ (m, 19H, Ar-H), 7.68 (d, $J=7.8$ $\mathrm{Hz}, 2 \mathrm{H}, \mathrm{Ar}-\mathrm{H}), 8.11$ (d, $J=9.3 \mathrm{~Hz}, 2 \mathrm{H}, \mathrm{Ar}-\mathrm{H}), 9.58(\mathrm{~s}, 1 \mathrm{H}$, $\mathrm{N}-\mathrm{H}) .{ }^{13} \mathrm{C}$ NMR $\left(75 \mathrm{MHz}, \mathrm{CDCl}_{3}\right) \delta 14.15,32.31,39.76$, $60.09,104.25,126.39,127,36,127.46,127.95,128.23,128.27$, $129.52,130.38,130.61,133.37,133.53,145.20,147.28,147.96$, $152.93,155.05,162.34,167.11$. IR (KBr) v 3304, 3081, 2980, 1687, 1634, 1505, 1353, 1096, 909, 770, $701 \mathrm{~cm}^{-1}$. HRMSFAB $m / z[\mathrm{M}+\mathrm{H}]^{+}$calcd for $\mathrm{C}_{47} \mathrm{H}_{39} \mathrm{~N}_{9} \mathrm{O}_{8} \mathrm{~S}_{2}: 921.24$. Found: 921.17. Anal. calcd for $\mathrm{C}_{47} \mathrm{H}_{39} \mathrm{~N}_{9} \mathrm{O}_{8} \mathrm{~S}_{2}: \mathrm{C}, 61.23 ; \mathrm{H}, 4.26 ; \mathrm{N}$, 13.67; S, 6.95. Found: C, 61.24; H, 4.28; N, 13.65; S, 6.94 .

\section{Conclusion}

In this study we demonstrated the synthesis, antibacterial and antifungal activity of new derivatives of 1,4-dihydropyridines bearing 1,2,4-triazole and 1,3,4-oxadiazole moieties at $\mathrm{C} 2$ and $\mathrm{C} 6$ of 1,4-DHP ring system. It should be noted that compound $\mathbf{8 d}$ exhibited the most potent activity against $E$. coli and $A$. fumigates. The structure-activity relationship of the compounds showed that substitution at the position 2, 6 of the pyridine ring enhances biological activity. Our results will have an impact on further investigation in this field in search of 1,4-dihydropyridine compounds connected with 1,2,4-triazole and 1,3,4-oxadiazole moieties as antibacterial and antifungal agents.
Acknowledgments. The authors are grateful to Urmia University for providing a fellowship for the present work and thanks are also given to Prof. Dr. Joachim Thiem from Hamburg University for the ESI-MS measurements.

\section{References}

1. D. J. Triggle, Biochem. Pharmacol. 2007, 74, 1-9. DOI:10.1016/j.bcp.2007.01.016

2. D. A. Sica, J. Hypertens. 2006, 8, 53-56.

3. F. Bossert, H. Meyer, E. Wehinger, Angew. Chem. Int. Ed. Engl. 1981, 20, 762-769.

DOI:10.1002/anie.198107621

4. J. G. Breitenbucher, G. Figliozz, Tetrahedron Lett. 2000, 41, 4311-4315. DOI:10.1016/S0040-4039(00)00660-2

5. G. A. Wachter, M. C. Davis, J. Med. Chem. 1998, 41, 24362438. DOI:10.1021/jm 9708745

6. T. Akbarzadeh, S. Motagian, A. Fallah Tafti, A. Shafiee, A. R. Shahverdi, Res. Pharm. Sci. 2008, 3, 87-93.

7. S. Kalam, B. Darna, A. Garlapati, M. R. Vanga, Eur. J. Med. Chem. 2011, 46, 1564-71.

DOI:10.1016/j.ejmech.2011.02.003

8. V. M. Briukhanov, Exp. Clin. Pharmocol, 1994, 57, 47-49.

9. R. Boer, V. Gekeler, Drugs Future, 1995, 20, 499-509.

10. O. Firuzi, K. Javidnia, E. Mansourabadi, L. Saso, A. R. Mehdipour, R. Miri, Arch. Pharm. Sci. Res. 2013, 36, 1392-1402. DOI:10.1007/s12272-013-0149-8

11. S. Ulloora, S. Kumar, R. Shabaraya, A.V. Adhikari, Med. Chem. Res, 2013, 22, 1549-1562.

DOI:10.1007/s00044-012-0156-1

12. G. Hamilton, E. P. Cosentini, B. Teleky, T. Koperna, J. Zacheri, M. Riegler, W. Feil, R. Schiessel, E. Wenzi, Anticancer Res. 1993, 13, 2059-2063.

13. H. W. van Veen, R. Callaghan, L. Soceneantu, A. Sardini, W. N. Konings, C. F. Higgins, Nature 1998, 391, 291-295. DOI: $10.1038 / 34669$

14. N. B. Patel, J. C. Patel, Sci. Pharm. 2010, 78, 171-193. DOI:10.3797/scipharm.0912-16

15. T. Plech, M. Wujec, A. Siwek, U. Kosikowska, A. Malm, Eur. J. Med. Chem. 2011, 46, 241-248.

DOI:10.1016/j.ejmech.2010.11.010

16. O. Prakash, M. Kumar, R. Kumar, C. Sharma, K. R. Aneja, Eur. J. Med. Chem. 2010, 45, 4252-4257.

DOI:10.1016/j.ejmech.2010.06.023

17. T. Tsukuda, Y. Shiratori, M. Watanabe, H. Ontsuka, K. Hattori, M. Shirai, Bioorg. Med. Chem. Lett. 1998, 8, 1819-1824. DOI:10.1016/S0960-894X(98)00316-3

18. M. A. Alia, M. Shaharyar, Bioorg. Med. Chem. Lett. 2007, 17, 3314-3316. DOI:10.1016/j.bmcl.2007.04.004

19. T. A. Kaplancikli, G. Turan-Zitouni, P. Chevallet, J.Enzyme. Inhib.Med.Chem. 2005, 20, 179-182.

DOI:10.1080/14756360500043471

20. K. Liu, X. Lu, H. J. Zhang, J. Sun, H. L. Zhu, Eur. J. Med. Chem. 2012, 47, 473-478. DOI:10.1016/j.ejmech.2011.11.015

21. A. Kamal, N. Shankaraiah, V. Devaiah, K. L. Reddy, A. Ju- 
vekar S. Sen, N. Kurian, S. Zingde, Bioorg. Med. Chem. Lett. 2008, 18, 1468-1473. DOI:10.1016/j.bmcl.2007.12.063

22. A. Kumar, C. S. Rajput, Eur. J. Med. Chem. 2009, 44, 83-90. DOI:10.1016/j.ejmech.2008.03.018

23. H. Kumar, S. A. Javed, S. A. Khan. Eur. J. Med. Chem. 2008, 43, 2688-2698. DOI:10.1016/j.ejmech.2008.01.039

24. R. R. Somani, G. Kadam, R. Vohra, S. Vijayaraghavan, P. Y. Shirodkar, Int. J. Pharmacol. 2010, 6, 696-704. DOI:10.3923/ijp.2010.696.704

25. N. Siddiqui, W. Ahsan, Eur. J. Med. Chem. 2010, 45, 15361543. DOI:10.1016/j.ejmech.2009.12.062

26. R. Surendrakumar, A. Manilal, A. J. Abdul Nasser, B. Merdekios, X. Chen, A. Idhayadhulla, J. Pharmacol. Toxicol. 2014, 9, 119-128. DOI:10.3923/jpt.2014.119.128

27. A. B. Archana, D. R. Dinesh, S. G. Paraag, Y. Shirodkar Prabhakar, Int. J. Pharm. Chem. 2014, 4, 63-67.

28. A. Hantzsch, Ber. 1881, 14, 1637-1638. DOI: $10.1002 /$ cber. 18810140214

29. A. Hantzsch, Ann. Chem. 1892, 215, 1-81. DOI:10.1002/jlac.18822150102

30. K. Akbari Dilmaghani, F. Nasuhi Pur, N. Hoseini Jazani, A. Alavi, Z. Niknam, F. Mirfakahraee, Phosphorus, Sulfur Silicon Relat. Elem.2014, 189, 81-87. DOI:10.1080/10426507.2013.789877

31. K. Akbari Dilmaghani, F. Nasuhi Pur, M. Hatami Nezhad, Iran. J. Pharm. Res. 2015, 14, 693-699.
32. K. Akbari Dilmaghani, F. Nasuhi Pur, M. Mahammadpour, J. Mahammadnejad, Iran. J. Pharm. Res. 2016, 15,777-782.

33. A. Tukmechi A. M. Ownagh, Braz. J. Microbiol. 2010, 41, 1086-1092. DOI:10.1590/S1517-83822010000400030

34. H. Katiroglu, Y. Beyatli, B. Aslim, Z. Yüksekdag, T. Atici, Internet J. Microbiol.2006, 2, 520-524.

35. N. K. Singh, R. J. Butcher, P. Tripathi, A. K. Srivastava. M. Kumar Bharty, Acta Cryst. 2007, E63, 0782- 0784.

36. C. H. Lee, H. I. Cho, K. J. Lee, Bull. Korean Chem. Soc. 2001, 22, 1153-1155.

37. M. Mhasalkar, M. Shah, S. Nikam, K. Anantanarayanan, Deliwala, J. Med. Chem. 1970, 13, 672-674.

DOI:10.1021/jm00298a021

38. I. Khan, S. Ali, S. Hameed, N. Rama, M. Hussain, A. Wadood, R. Uddin, Z. Ul-Haq, A. Khan, S. Ali, M. Choudhary, Eur. J. Med. Chem. 2010, 45, 5200-5207.

DOI:10.1016/j.ejmech.2010.08.034

39. (a) B. V. I. Cohen, J. Heterocycl. Chem. 1978, 15, 237-240. DOI:10.1002/jhet.5570150211

(b) K. Sung, A. R. Lee, J. Heterocycl. Chem. 1992, 29, 11011109. DOI:10.1002/jhet.5570290512

40. M. S. Bai, Y. Y. Chen, D. L. Niu, L. Peng, Acta Cryst. 2009, E65, 0799.

41. M. Petrova, R. Muhamadejev, B. Vigante, B. Cekavicus, A. Plotniece, G. Duburs, E. Liepinsh, Molecules 2011, 16, 80418052. DOI:10.3390/molecules 16098041

\section{Povzetek}

Pripravili smo serijo dietil-2,6-dimetil-4-fenil-1,4-dihidropiridin-3,5-dikarboksilatnih derivatov spojenih z 1,3,4-oksadiazol-5-tionskimi in 1,2,4-triazol-5-tionskimi ostanki na položajih C2,C6 v 1,4-dihidropiridinskem obročnem sistemu. To povezavo smo izvedli z reakcijo med 1,3,4-oksadiazol-5-tioni in 1,2,4-triazol-5-tioni z 2,6-dibromometil-3,5-dietoksikarbonil-4-fenil-1,4-dihidropiridinom ob prisotnosti kalijevega karbonata kot šibke baze in v suhem acetonu kot topilu. Nove pripravljene spojine smo karakterizirali z FT-IR, ${ }^{1} \mathrm{H}$ NMR in ${ }^{13} \mathrm{C}$ NMR spektroskopskimi podatki, kot tudi z elementno analizo in FAB-MS. Za sintetizirane spojine smo in vitro preverili delovanje proti mikrobom in glivam (proti Escherichia coli in Aspergillus fumigatus) ter rezultate primerjali z vrednostmi za enrofloksacin in amfotericin kot referenčnima zdraviloma, ki se običajno uporabljata za zdravljenje tovrstnih infekcij. Pripravljene spojine so izkazale različne inhibicijske sposobnosti proti testiranim bakterijam in glivam. Spojina 8d je pokazala največje antagonistične lastnosti proti E. coli and A. fumigatus. 